\title{
The Association between Angiotensin II Type 1 Receptor Gene A1166C Polymorphism and Non-alcoholic Fatty Liver Disease and Its Severity
}

\author{
Ahad Eshraghian ${ }^{1,2, *}$, Shahrokh Iravani ${ }^{1}$, Pedram Azimzadeh ${ }^{3}$
}

1. Department of Gastroenterology and Hepatology, AJA University of Medical Sciences, Tehran, Iran

2. Gastroenterohepatology Research Center, Shiraz University of Medical Sciences, Shiraz, Iran

3. Research Institute for Gastroenterology and Liver Diseases, Shahid Beheshti University of Medical Sciences, Tehran, Iran

\section{* Corresponding Author:}

Ahad Eshraghian, MD

Gastroenterohepatology Research Center, Nemazee Hospital, Shiraz, Iran PO Box: 71937-11351, Shiraz, Iran Telefax: + 987136281442 Email: Eshraghiana@yahoo.com

Received: 03 Dec. 2017

Accepted: 20 Mar. 2018

\section{ABSTRACT}

\section{BACKGROUND}

Genetic predisposition may have important role in pathogenesis of non-alcoholic fatty liver disease (NAFLD). Angiotensin II type I receptor (AGTR1) has been known to involve in the process of liver steatosis and fibrosis. This study aimed to investigate the association between AGTR1 A1166C polymorphism and NAFLD.

\section{METHODS}

A cross-sectional study was conducted during May 2014-May 2015 among healthy adults referring to our radiology clinic for abdominal sonography. AGTR1 A1166C polymorphism was evaluated in subjects with NAFLD and healthy individuals using allelic discrimination method.

\section{RESULTS}

58 subjects with NAFLD were compared with 88 healthy individuals without NAFLD. The frequency of AA and CC genotypes of AGTR1 was significantly higher in patients with NAFLD compared with controls ( $p=0.029$ and 0.042 , respectively). C allele was more detected in subjects with NAFLD compared with the healthy controls (OR: $2.1 ; 95 \%$ CI: $1.23-3.61, p=0.006$ ). CC genotype (OR: $10.62 ; 95 \%$ CI: $1.05-106.57, p=0.045)$ and C allele (OR: 6.81; 95\% CI: 1.42 $32.48, p=0.016)$ were also predictors of severe fatty liver disease in our study population.

\section{CONCLUSION}

Our results provide the first evidence that AGTR1 gene A1166C polymorphism not only is associated with NAFLD and but also may predict its severity.

\section{KEYWORDS:}

Non-alcoholic fatty liver disease, Non-alcoholic steatohepatitis, Metabolic syndrome, AGTR1 gene A1166C polymorphism

Please cite this paper as:

Eshraghian A, Iravani S, Azimzadeh P. The Association between Angiotensin II Type 1 Receptor Gene A1166C Polymorphism and Non-alcoholic Fatty Liver Disease and Its Severity. Middle East J Dig Dis 2018;10:96-104. doi: 10.15171/mejdd.2018.97.

\section{INTRODUCTION}

Non-alcoholic fatty liver disease (NAFLD) is usually considered as the hepatic feature of metabolic syndrome and is a rapidly growing disease worldwide. ${ }^{1}$ NAFLD is a clinical spectrum ranging from simple steatosis to non-alcoholic steatohepatitis (NASH), which may progress to liver cirrhosis and hepatocellular carcinoma. ${ }^{2}$ NAFLD is now considered as the most common cause of abnormalities in liver function tests. However, its early diagnosis is important because of its potential to progress to liver cirrhosis and hepatocellular carcinoma. ${ }^{3}$ 


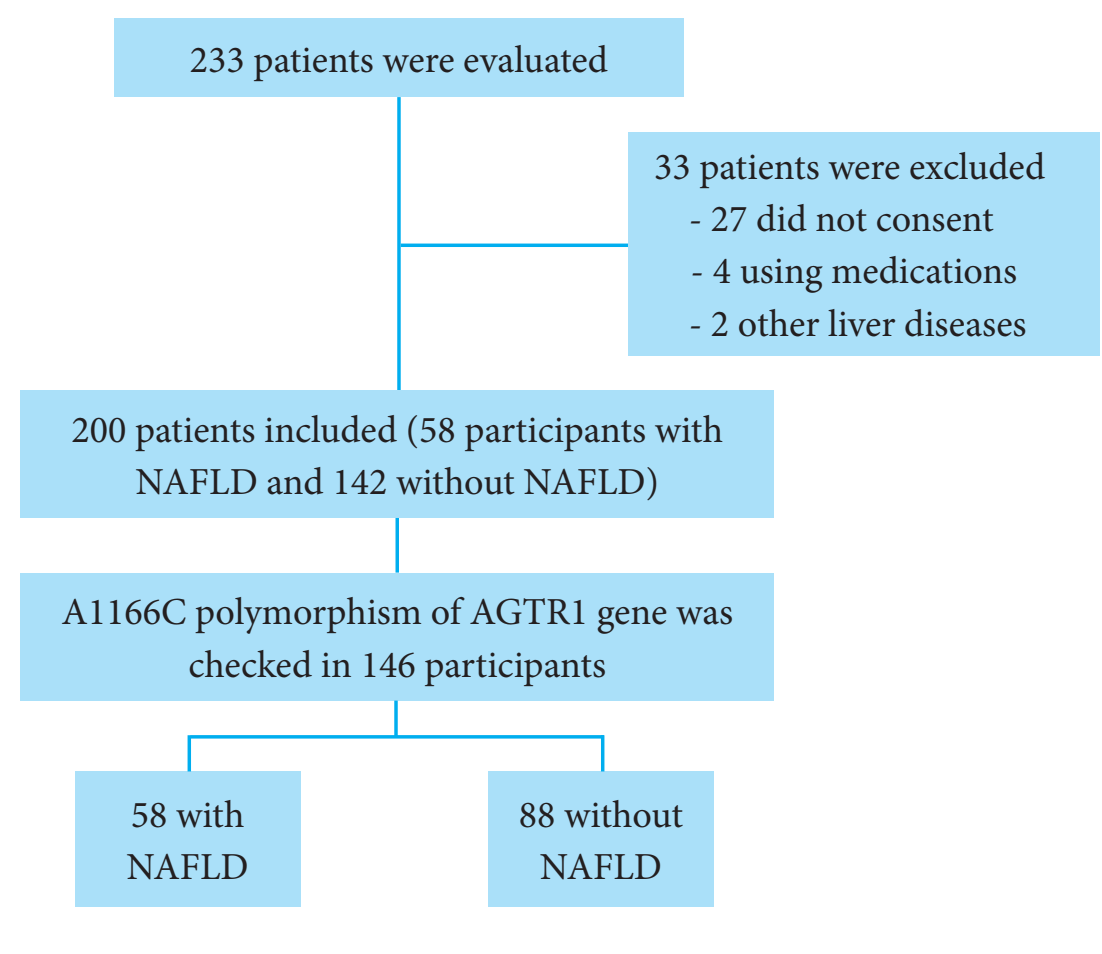

Fig.1: Flow Diagram of the study

Many hepatologists now believe that NAFLD/NASH is an important cause of liver cirrhosis in patients marked as cryptogenic cirrhosis. ${ }^{4}$ Furthermore, there is substantial evidence suggesting the association between NAFLD and sub-clinical atherosclerosis independent of traditional metabolic risk factors. ${ }^{5}$ Therefore, understanding the pathogenesis, risk factors, and new treatment modalities of NAFLD/NASH can be an important priority in the field of hepatology. Some traditional risk factors for NAFLD include metabolic syndrome, obesity, hyperlipidemia, advanced age, and insulin resistance. ${ }^{6}$ Subsequently, the global increase in the prevalence of NAFLD is probably secondary to global increase in metabolic syndrome and obesity, however, the role of genetic predisposition to NAFLD/NASH should not be neglected. ${ }^{7}$ NAFLD can occur in the absence of metabolic syndrome and its components and this especially highlights the role of genetic susceptibility as an important stimulus of the disease. ${ }^{8}$

Renin-angiotensin system (RAS) is an enzymatic pathway participated in the regulation of blood pressure, cardiovascular system, and electrolyte balance. ${ }^{9}$ Liver is the primary source of angiotensinogen that is converted to angiotensin I by rennin. Thereafter, angiotensin I is converted to angiotensin II by angiotensin converting enzyme (ACE).$^{10}$ Angiotensin II acts through two Gprotein-coupled receptor subtypes: type 1 angiotensin II type 1 receptor (AGTR) and type 2 receptor. ${ }^{10}$ Genetic polymorphisms of AGTR1 have been suggested to be associated with cardiovascular disease and metabolic syndrome. ${ }^{11}$ In animal studies, the deletion of AGTR1 was associated with diffuse hepatic steatosis. ${ }^{12}$ Furthermore, AGTR1 interactions have been observed in promotion of hepatic fibrosis. ${ }^{13}$ Such evidence suggest AGTR1 role as an important regulator of hepatic steatosis. This study aimed to investigate the association between polymorphisms of AGTR1 with NAFLD and its metabolic components in a cluster of Iranian population.

\section{MATERIALS AND METHODS}

A cross-sectional study was conducted during MayOctober 2014 among healthy adults (aged $>18$ years) referring to our radiology clinic for abdominal sonography. (Figure 1) NAFLD was diagnosed according to below mentioned sonographic criteria. An expert radiologist with enough expertise in diagnosis of fatty liver performed all ultrasound procedures. The radiologist was totally un- 
aware of clinical and laboratory tests of the participants. Participants with a history of liver cirrhosis, underlying liver diseases such as autoimmune hepatitis, hepatitis B or $\mathrm{C}$ viruses induced hepatitis, Wilson's disease, hepatobiliary cancers, those with $>20 \mathrm{~g} /$ day alcohol consumption, and individuals receiving some specific medications known to cause hepatic steatosis (like amiodarone, valproic acid, etc) were excluded from the study. Finally, the patients with NAFLD were compared with those without NAFLD.

A data gathering form was used for recording the participants' characteristics including sex, age, height, weight, body mass index (BMI), hip circumference, waist circumference, waist-to-hip ratio, systolic and diastolic blood pressure, and a history of hyperlipidemia, diabetes, hypertension, and ischemic heart disease (IHD). BMI was calculated using this formula: body weight $(\mathrm{kg}) /$ square of height $\left(\mathrm{m}^{2}\right)$. A well trained examiner measured waist circumference at the midpoint between the lower costal margin and the anterior superior iliac crest using a standard tape. Hip circumference was measured at the widest point between the hip and the buttock by the examiner. Systolic and diastolic blood pressure of brachial artery was measured using appropriate cuffs. Blood samples $(10 \mathrm{~mL})$ were drawn in standard tubes and were sent to our research lab affiliated to Shahid Beheshti University of Medial Sciences, Tehran, Iran. Serum aspartate aminotransferase (AST), alanine aminotransferase (ALT), alkaline phosphatase (ALP), albumin, total cholesterol, high-density lipoprotein (HDL), triglyceride, low density lipoprotein (LDL), and glucose were measured using the same commercial kits with standard biochemical chemistry methods.

\section{Definition}

NAFLD was diagnosed based on ultrasonographic findings. These four criteria were used for diagnosis of hepatic steatosis in ultrasonography: hepatorenal echo contrast, liver brightness, deep attenuation, and vascular blurring. The severity of liver echogenicity was considered as a marker of severity of liver steatosis and classified as below: grade 0: normal echogenicity; grade 1: normal visualization of diaphragm and intrahepatic vessel borders but slight, diffuse increase in fine echoes in liver parenchyma; grade 2: moderate, slightly impaired visualization of intrahepatic vessels and diaphragm with diffuse increase in fine echoes of liver parenchyma; grade 3: marked increase in the fine echoes of liver parenchyma with poor or non-visualization of the intrahepatic vessel borders, diaphragm, and posterior right lobe of the. ${ }^{14}$ NAFLD was defined as the presence of above mentioned ultrasound criteria and absence of chronic liver diseases such as autoimmune hepatitis, hepatitis $\mathrm{B}$ or $\mathrm{C}$ viruses induced hepatitis, hepatobiliary cancers, Wilson's disease, $>10$ $\mathrm{g}$ /day alcohol consumption, and receiving some specific medications known to cause hepatic steatosis (like amiodarone, valproic acid, etc).

National Cholesterol Education Program and Adult Treatment Panel III (NCEP: ATPIII) criteria were used for definition of metabolic syndrome. Metabolic syndrome was defined as the presence of three or more of the following metabolic components: 1: Central obesity as waist circumference $>102 \mathrm{~cm}$ for men and $>88 \mathrm{~cm}$ for women, 2: Hypertriglyceridemia as triglyceride $\geq 150$ $\mathrm{mg} / \mathrm{dL}$ or taking specific medications, 3: Low HDL cholesterol as $<40 \mathrm{mg} / \mathrm{dL}$ in men and $<50 \mathrm{mg} / \mathrm{dL}$ in women or specific medications, 4: Systolic blood pressure $\geq 130$ $\mathrm{mmHg}$ or diastolic blood pressure $\geq 85 \mathrm{mmHg}$ or specific medications, and 5: Fasting plasma glucose $\geq 100 \mathrm{mg} / \mathrm{dL}$ or specific medications or previously diagnosed type II diabetes. ${ }^{15}$ An ALT level over 40 IU/L was considered abnormal. Diabetes mellitus was defined as having fasting plasma glucose $\geq 126 \mathrm{mg} / \mathrm{dL}$ or taking glucose lowering agents. The participants were considered to have high blood pressure if they used anti-hypertensive medications or if they had systolic blood pressure $\geq 140 \mathrm{~mm} / \mathrm{Hg}$ or diastolic blood pressure $\geq 90 \mathrm{~mm} / \mathrm{Hg}$. The sample size was estimated assuming a prevalence of $25 \%$ for a $95 \%$ confidence interval and $80 \%$ power.

\section{Genotyping}

Whole blood was collected from the study participants in EDTA tubes and the genomic DNA was extracted from the peripheral blood leukocytes using a QIAamp DNA Blood Kit (QIAGEN Inc., Hilden, Germany). DNA was stored at $-20^{\circ} \mathrm{C}$ until the time of usage. TaqMan polymerase chain reaction (PCR) was applied for determination of $\mathrm{A} \rightarrow \mathrm{C}$ transversion at nucleotide position 1166 of the angiotensin II type 1 receptor gene (AGTR1 A1166C). Genotype determination was performed using 
Table 1: Baseline characteristics of the study participants

\begin{tabular}{|c|c|c|c|}
\hline Variables & With NAFLD (58 patients) & Without NAFLD (142 patients) & $P$ value \\
\hline Age (year) & $40.71 \pm 6.84$ & $37.07 \pm 7.03$ & 0.0001 \\
\hline Weight (kg) & $83.46 \pm 9.73$ & $77.44 \pm 7.57$ & 0.0001 \\
\hline Height $(\mathrm{cm})$ & $172.53 \pm 6.27$ & $174.76 \pm 5.67$ & 0.015 \\
\hline BMI $\left(\mathrm{kg} / \mathrm{m}^{2}\right)$ & $28.06 \pm 3.23$ & $25.35 \pm 2.21$ & 0.0001 \\
\hline Waist circumference $(\mathrm{cm})$ & $87.75 \pm 7.42$ & $83.67 \pm 7.24$ & 0.0001 \\
\hline Hip circumference $(\mathrm{cm})$ & $101.06 \pm 4.70$ & $96.03 \pm 4.56$ & 0.0001 \\
\hline Triglyceride (mg/dL) & $183.37 \pm 73.57$ & $161.62 \pm 42.29$ & 0.15 \\
\hline HDL (mg/dL) & $41.50 \pm 5.54$ & $42.05 \pm 5.28$ & 0.50 \\
\hline $\mathrm{LDL}(\mathrm{mg} / \mathrm{dL})$ & $133.98 \pm 15.99$ & $129.30 \pm 18.86$ & 0.09 \\
\hline Cholesterol (mg/dL) & $199.10 \pm 44.09$ & $184.07 \pm 38.76$ & 0.018 \\
\hline $\mathrm{FPG}(\mathrm{mg} / \mathrm{dL})$ & $88.60 \pm 16.15$ & $86.95 \pm 10.89$ & 0.40 \\
\hline ALT (IU/L) & $38.56 \pm 17.61$ & $20.76 \pm 6.40$ & 0.0001 \\
\hline AST (IU/L) & $23.98 \pm 8.97$ & $22.92 \pm 6.37$ & 0.34 \\
\hline ALP (IU/L) & $159.96 \pm 43.46$ & $160.90 \pm 33.70$ & 0.87 \\
\hline $\operatorname{Albumin}(\mathrm{mg} / \mathrm{dL})$ & $4.33 \pm 0.43$ & $4.38 \pm 0.36$ & 0.37 \\
\hline
\end{tabular}

NAFLD: Non-alcoholic fatty liver disease; BMI: body mass index; LDL: Low density lipoprotein; HDL: high density lipoprotein; FPG: fasting plasma glucose; ALT: alanine aminotransferase; ALP: Alkaline phosphatase; AST: Aspartate aminotransferase; kg: kilogram; cm: centimeter

TaqMan 5' nuclease assay (Applied Biosystems, Foster City, CA) by two TaqMan probes, one for each allele. Allelic Discrimination (AD) method and applied biosystem (ABI) Proteomic Investigation Strategy for Mammals (PRISM) 7500 instrument and sequence detector software (SDS) 1.1 (Applied Biosystems, Foster City, CA) were applied for data analysis and genotyping. Genotyping PCR reaction consisted of TaqMan Universal PCR Master Mix 2X, TaqMan predesigned primers, and probes mixture (assay ID C_441625_10, Applied Biosystems, Foster City, CA), deionized water, and $20 \mathrm{ng}$ of purified genomic DNA.PCR was performed at $93^{\circ} \mathrm{C}$ for $5 \mathrm{~min}$, followed by 30 cycles at $93^{\circ} \mathrm{C}$ for $60 \mathrm{~s}$, at $55^{\circ} \mathrm{C}$ for $60 \mathrm{~s}$ and $72^{\circ} \mathrm{C}$ for $60 \mathrm{~s}$. A final extension step was carried out at $72^{\circ} \mathrm{C}$ for $5 \mathrm{~min}$.

\section{Ethics and consent}

Prior approval for the sonography and blood sampling were obtained from the Ethics Committee of AJA University of Medical Sciences, Tehran, Iran. A written informed consent including all steps of the study, the possible harms and benefits of the study were signed by all the participants after careful description of the study protocol. The study was performed in accordance with the Helsinki Declaration as revised in Seoul in 2008.

\section{Statistical analysis}

Student's t test was used for comparisons of continuous variables. Chi-square test was used for analysis of categorical variables. Mann-Whitney test (non-parametric test) was used when appropriate. Logistic regression model was applied to identify independent risk factors of NAFLD. SPSS software version 16.0 (SPSS Inc.; Chicago, IL, USA) was used for statistical analysis. P values $<0.05$ were considered as statistically significant.

\section{RESULTS}

Of 233 individuals, 33 subjects were excluded from the study ( 27 did not consent to participate in the study, 4 due to medications, and 2 due to other causes of liver disease). Of the remaining 200 individuals who completed the study, 58 (29\%) subjects were found to have different degrees of fatty liver. We categorized the severity of fatty infiltration of the liver (based on sonographic findings) to mild, moderate, and severe. In patients with NAFLD, 31 (15.5\%) subjects had mild NAFLD, and 27 (13.5\%) had moderate to severe NAFLD. Mean age of the patients with NAFLD was $40.71 \pm 6.84$ years compared with those without NAFLD $37.07 \pm 7.03$ years $(p=000.1)$. Baseline characteristics and anthropometric indices of the study participants were outlined in table 1. Mean of all anthropometric 
Table 2: Associated risks in subjects with NAFLD and those without NAFLD

\begin{tabular}{lcccc}
\hline Variables & NAFLD $(+)$ & NAFLD $(-)$ & OR $(\mathbf{9 5 \%}$ CI) & $P$ value \\
\hline Metabolic Sy & $31(53.44 \%)$ & $27(19.01 \%)$ & $3.51(1.84-6.66)$ & $<0.001$ \\
\hline DM & $6(10.34 \%)$ & $4(2.81 \%)$ & $3.2(1.58-9.71)$ & $<0.01$ \\
\hline HLP & $15(25.86 \%)$ & $12(8.45 \%)$ & $3.77(1.64-8.69)$ & 0.002 \\
\hline CAD & $2(3.44 \%)$ & $1(0.7 \%)$ & $5.03(0.44-10.66)$ & 0.20 \\
\hline HTN & $3(5.17 \%)$ & $1(1.4 \%)$ & $7.61(0.78-15.74)$ & 0.1 \\
\hline Smoking & $9(15.51 \%)$ & $13(9.15 \%)$ & $1.82(0.73-4.53)$ & 0.14 \\
\hline
\end{tabular}

NAFLD: Non-alcoholic fatty liver disease; Metabolic Sy: Metabolic syndrome; DM: Diabetes mellitus; HLP: Hyperlipidemia; HTN: Hypertension; CAD: Coronary artery disease; OR Odds Ratio; CI: Confidence interval

Table 3: Multivariate regression analysis for the NAFLD predictors

\begin{tabular}{lccc}
\hline Variables & OR & $\mathbf{( 9 5 \% ~ C I )}$ & $\boldsymbol{P}$ value \\
\hline BMI & 7.74 & $(1.25-3.73)$ & 0.005 \\
\hline Waist circumference & 9.26 & $(0.68-0.92)$ & 0.002 \\
\hline Hip circumference & 5.21 & $(1.02-1.39)$ & 0.022 \\
\hline Metabolic syndrome & 15.21 & $(9.74-14.43)$ & $<0.001$ \\
\hline ALT & 26.46 & $(1.19-1.50)$ & $<0.001$ \\
\hline
\end{tabular}

NAFLD: Non-alcoholic fatty liver disease; BMI: Body mass index; ALT: Alanine aminotransferase; OR: Odds Ratio; CI: Confidence interval

Table 4: Association between A1166C polymorphism of AGTR1 gene and NAFLD

\begin{tabular}{|c|c|c|c|c|c|}
\hline Variables & NAFLD (+) & NAFLD (-) & OR & $(95 \%$ CI $)$ & $P$ value \\
\hline \multicolumn{6}{|l|}{ Genotype } \\
\hline $\mathrm{AA}$ & $25(43.1 \%)$ & $57(64.7 \%)$ & 1 & Reference & 1 \\
\hline $\mathrm{AC}$ & $27(46.5 \%)$ & $28(31.8 \%)$ & 2.19 & $1.08-4.46$ & 0.029 \\
\hline $\mathrm{CC}$ & $6(10.3 \%)$ & $3(3.4 \%)$ & 4.56 & $1.05-19.70$ & 0.042 \\
\hline \multicolumn{6}{|l|}{ Allele } \\
\hline A & $77(66.3 \%)$ & $142(80.6 \%)$ & 1 & Reference & 1 \\
\hline $\mathrm{C}$ & $39(33.6 \%)$ & $34(19.3 \%)$ & 2.11 & $1.23-3.61$ & 0.006 \\
\hline
\end{tabular}

NAFLD: Non-alcoholic fatty liver disease; OR: Odds Ratio; CI: Confidence interval

indices including BMI, weight, height, waist circumference, and hip circumference were significantly higher in subjects with NAFLD compared with those without NAFLD $(p<0.05$, table 1$)$. Mean total cholesterol was significantly higher in subjects with NAFLD in comparison with the controls using univariate analysis ( $p=$ 0.018 , table1). Mean HDL, LDL, triglyceride, and fasting plasma glucose were not associated with NAFLD in univariate analysis (table 1). Higher serum ALT was also a predictor of NAFLD (38.56 \pm 17.61 vs. $20.76 \pm$ $6.40 \mathrm{IU} / \mathrm{L})(p=0.0001)$. Metabolic syndrome was detected in 31 (53.44\%) individuals in NAFLD group and in $27(19.01 \%)$ individuals in the control group ( $p<$ 0.001) (OR: 3.51, 95\% CI: 1.84-6.66). Personal history of hyperlipidemia and diabetes mellitus were also asso- ciated with NAFLD $(p<0.05$, table 2$)$.

Multivariate logistic regression analysis of risk factors showed that BMI, metabolic syndrome, waist circumference, hip circumference, and serum ALT were independent predictors of NAFLD in our study population (table 3 ).

58 subjects with NALFD were compared with $88 \mathrm{sub}$ jects without NAFLD regarding A1166C polymorphism of AGTR1 gene. The genotype frequencies of the gene polymorphisms did not significantly differ from the values predicted by Hardy-Weinberg equilibrium. The frequencies of $\mathrm{AA}, \mathrm{AC}$, and $\mathrm{CC}$ genotypes were outlined in table 4 . The AC and $\mathrm{CC}$ genotypes were significantly associated with NAFLD in our study population (table 4). $\mathrm{C}$ allele was more detected in subjects with NAFLD compared with the healthy controls (OR: 2.1; 95\% CI: 


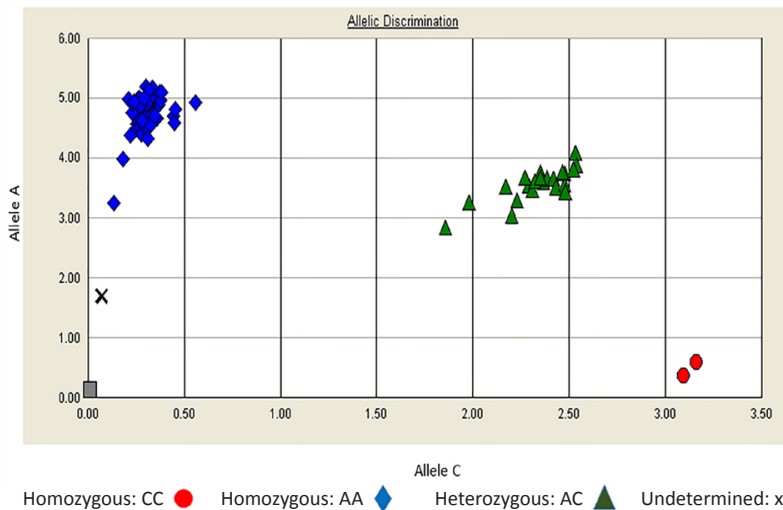

Fig.2: Allelic discrimination plot for $\mathrm{A} 1166 \mathrm{C}$ polymorphism of AGTR1

1.23-3.61, $p=0.006)$. The association between disease severity and frequencies of genotypes and alleles were also determined. CC genotype (OR: 10.62; 95\% CI: $1.05-106.57, p=0.045)$ and $\mathrm{C}$ allele (OR: $6.81 ; 95 \% \mathrm{CI}$ : $1.42-32.48, p=0.016)$ were also predictors of severe fatty liver disease in our study population.

\section{DISCUSSION}

The prevalence of NAFLD irrespective of severity was $29 \%$ in our study population. However, only $13.5 \%$ had moderate to severe fatty liver based on ultrasound findings. This study population was totally composed of citizens of a large city, Shiraz, with modern life styles. This prevalence is comparable to the previous report from this region. ${ }^{16-18}$

Results of the present study showed that BMI, metabolic syndrome, waist circumference, hip circumference (markers of central obesity), and serum ALT were all associated with the presence of NAFLD independently. These findings were also confirmed the results of the previous studies from this region. ${ }^{19}$ NAFLD has no specific heralding sign or symptoms and many patients are discovered in routine health examinations. ${ }^{20}$ Therefore, in obese individuals and in subjects with central obesity especially with high serum ALT level, careful evaluation and follow-up should be considered for early detection and treatment of NAFLD.

Two previous studies confirmed the association between other AGTR1 gene polymorphisms and NAFLD. ${ }^{21,22}$ However, the results of our study provide the first evidence of the association between AGTR1 gene A166C polymorphism and NAFLD. AC and CC genotypes as well as $\mathrm{C}$ allele were predictors of NAFLD in our study population. The other novel finding was the association between CC genotype and C allele of AGTR1 gene with more severe forms of NAFLD. (Figure 2)

The role of rennin-angiotensin system in liver fibrosis and steatosis has been previously investigated..$^{12}$ Granzow and colleagues showed that AGTR1 mediated activation of Janus kinase-2 (JAK-2) led to activation of hepatic stellate cells (HSCs) and liver fibrosis. ${ }^{23}$ Angiotensin II also facilitates fibrogenic effects of transforming growth factor- $\beta$ (TGF- $\beta$ ) and promotes liver fibrosis. ${ }^{24}$ Other study on a rat model of NASH showed that development of hepatic fibrosis in NASH might be the result of interaction between toll like receptor-4 (TLR-4) and angiotensin II. ${ }^{25}$ Based on these findings some authors started to investigate the role of blocking rennin-angiotensin system in the treatment of NAFLD/NASH in animal models, ${ }^{26,27}$ and in humans..$^{28,29}$

A1166C polymorphism of AGTR1 has been studied in some studies of NAFLD. In Iranian population, a recent study confirmed the association between A1166C polymorphism of AGTR1 gene and diabetes mellitus type 2 (DM II) and coronary artery disease. C allele was also more frequently detected in patients with DM $\mathrm{II}$ and coronary artery disease. ${ }^{30}$ Palatini and colleagues showed that $\mathrm{C}$ allele of A1166C polymorphism of AGTR1 predicted hypertension and metabolic syndrome. ${ }^{31} \mathrm{An}$ other previously published study among patients with metabolic syndrome has also confirmed these results. ${ }^{32}$ CC genotype of AGTR1 gene A1166C was reported to be associated with central obesity and dyslipidemia. ${ }^{33}$ Frequency of $\mathrm{C}$ allele in AGTR1 gene A1166C was also reported to be higher in subjects with insulin resistance as a strong suggested underlying mechanism for hepatic steatosis. ${ }^{34}$ Such substantial evidence and results of the present study all signify the role of RAS and AGTR1 gene A1166C polymorphism in the pathogenesis of NAFLD.

Adiponectin is an adipocytokine that is expressed in visceral adipose tissue and is involved in energy and fat metabolism. Increased adiponectin level seems to be protective against insulin resistance and decreased plasma adiponectin has been observed in DM II and cardiovascular disease. ${ }^{35-37}$ Plasma adiponection level is 
also decreased in patients with NAFLD. ${ }^{38}$ On the other hand, there is a direct association between plasma adiponection level and rennin angiotensin system though that blockade of rennin-angiotensin system causes increase in serum adiponection level..$^{39}$

It has been previously suggested that $\mathrm{C}$ allele of AGTR1 A1166C has been associated with lower response to statin therapy. ${ }^{40}$ Statins have been shown to be safe and effective for the treatment of NASH especially in those with other risks for cardiovascular disease. ${ }^{41-43}$ Therefore, our findings are also important in clinical scenario and the association between AGTR1 gene A1166C and clinical response to treatment of NAFLD can be the topic for future studies. This study further emphasizes the important role of RAS in hepatic steatosis and supports targeting inhibition of renin-angiotensin pathway as a treatment option for NAFLD.

\section{ACKNOWLEDGEMENTS}

This study was supported partially by a grant from AJA University of Medical Sciences, Tehran, Iran.

\section{ETHICAL APPROVAL}

There is nothing to be declared.

\section{CONFLICT OF INTEREST}

The authors declare no conflict of interest related to this work.

\section{REFERENCES}

1. Vernon G, Baranova A, Younossi ZM. Systematic review: the epidemiology and natural history of nonalcoholic fatty liver disease and nonalcoholic steatohepatitis in adults. Aliment Pharmacol Ther 2011;34:274-85. doi: 10.1111/j.1365-2036.2011.04724.x.

2. Law K, Brunt EM. Nonalcoholic fatty liver disease. Clin Liver Dis 2010;14:591-604. doi: 10.1016/j. cld.2010.07.006.

3. Angulo P. GI epidemiology: nonalcoholic fatty liver disease. Aliment Pharmacol Ther 2007;25:883-9. doi: 10.1016/j.cld.2010.07.006.

4. Clark JM, Diehl AM. Nonalcoholic fatty liver disease: an under-recognized cause of cryptogenic cirrhosis. JAMA 2003;289:3000-4. doi: 10.1001/jama.289.22.3000.

5. Thakur ML, Sharma S, Kumar A, Bhatt SP, Luthra K, Guleria R, et al. Nonalcoholic fatty liver disease is associated with subclinical atherosclerosis independent of obesity and metabolic syndrome in Asian Indians. Ath- erosclerosis 2012;223:507-11. doi: 10.1016/j.atherosclerosis.2012.06.005

6. Eguchi Y, Hyogo H, Ono M, Mizuta T, Ono N, Fujimoto $\mathrm{K}$, et al. Prevalence and associated metabolic factors of nonalcoholic fatty liver disease in the general population from 2009 to 2010 in Japan: a multicenter large retrospective study. $J$ Gastroenterol 2012;47:586-95. doi: 10.1007/s00535-012-0533-Z.

7. Daly AK, Ballestri S, Carulli L, Loria P, Day CP. Genetic determinants of susceptibility and severity in nonalcoholic fatty liver disease. Expert Rev Gastroenterol Hepatol 2011;5:253-63. doi: 10.1586/egh.11.18.

8. Day CP. Genetic and environmental susceptibility to nonalcoholic fatty liver disease. Dig Dis 2010;28:255-60. doi: $10.1159 / 000282098$.

9. Kuba K, Imai Y, Ohto-Nakanishi T, Penninger JM. Trilogy of ACE2: a peptidase in the renin-angiotensin system, a SARS receptor, and a partner for amino acid transporters. Pharmacol Ther 2010;128:119-28. doi: 10.1016/j. pharmthera.2010.06.003.

10. Hanes DS, Nahar A, Weir MR. The tissue renin-angiotensin-aldosterone system in diabetes mellitus. Curr Hypertens Rep 2004;6:98-105.

11. Fung MM, Rao F, Poddar S, Mahata M, Khandrika S, Mahata SK, et al. Early inflammatory and metabolic changes in association with AGTR1 polymorphisms in prehypertensive subjects. Am J Hypertens 2011;24:22533. doi: 10.1038/ajh.2010.210.

12. Nabeshima Y, Tazuma S, Kanno K, Hyogo H, Chayama $\mathrm{K}$. Deletion of angiotensin II type I receptor reduces hepatic steatosis. J Hepatol 2009;50:1226-35. doi: 10.1016/j.jhep.2009.01.018.

13. Moreira de Macêdo S, Guimarães TA, Feltenberger JD, Sousa Santos SH. The role of renin-angiotensin system modulation on treatment and prevention of liver diseases. Peptides 2014;62:189-96. doi: 10.1016/j.peptides.2014.10.005.

14. Mishra P, Younossi ZM. Abdominal ultrasound for diagnosis of nonalcoholic fatty liver disease (NAFLD). Am $J$ Gastroenterol 2007;102:2716-7. doi: 10.1111/j.15720241.2007.01520.x.

15. Tan BY, Kantilal HK, Singh R. Prevalence of metabolic syndrome among Malaysians using the international diabetes federation, national cholesterol education program and modified World Health Organization definitions. Mal J Nutr 2008;1:65-77.

16. Eshraghian A. High Prevalence of Non-alcoholic Fatty Liver Disease in the Middle East: Lifestyle and Dietary Habits. Hepatology 2017;65:1077. doi: 10.1002/ hep. 28937.

17. Lankarani KB, Ghaffarpasand F, Mahmoodi M, Lotfi M, Zamiri N, Heydari ST, et al. Nonalcoholic fatty liver disease in southern Iran: a population based study. Hepat Mon 2013;13:e9248. doi: 10.5812/hepatmon.9248.

18. Sohrabpour A, Rezvan H, Amini-Kafiabad S, Dayhim 
M, Merat S, Pourshams A. Prevalence of Nonalcoholic Steatohepatitis in Iran: A Population based Study. Middle East J Dig Dis 2010;2:14-9.

19. Eshraghian A, Dabaghmanesh MH, Eshraghian H, Fattahi MR, Omrani GR. Non alcoholic fatty liver disease in a cluster of Iranian population: thyroid status and metabolic risk factors. Arch Iran Med 2013;16:64-7. doi: 10131610/AIM.007.

20. Friedman SL, Maher JJ, Bissell DM. Mechanisms and therapy of hepatic fibro-sis: report of the AASLD Single Topic Basic Research Conference. Hepatology 2000;32:1403-8. doi: 10.1053/jhep.2000.20243.

21. Zain SM, Mohamed Z, Mahadeva S, Rampal S, Basu RC, Cheah PL, et al. Susceptibility and Gene Interaction Study of the Angiotensin II Type 1 Receptor (AGTR1) Gene Polymorphisms with Non-Alcoholic Fatty Liver Disease in a Multi-Ethnic Population. PLoS One 2013;8:e58538. doi: 10.1371/journal.pone.0058538.

22. Yoneda M, Hotta K, Nozaki Y, Endo H, Uchiyama T, Mawatari $\mathrm{H}$, et al. Association between angiotensin II type 1 receptor polymorphisms and the occurrence of nonalcoholic fatty liver disease. Liver Int 2009;29:107885. doi: 10.1111/j.1478-3231.2009.01988.x.

23. Granzow M, Schierwagen R, Klein S, Kowallick B, Huss S, Linhart M, et al. Angiotensin-II type 1 receptormediated Janus kinase 2 activation induces liver fibrosis. Hepatology 2014;60:334-48. doi: 10.1002/hep.27117.

24. Li YS, Ni SY, Meng Y, Shi XL, Zhao XW, Luo HH, et al. Angiotensin II facilitates fibrogenic effect of TGF- $\beta 1$ through enhancing the down-regulation of BAMBI caused by LPS: a new pro-fibrotic mechanism of angiotensin II. PLoS One 2013;8:e76289. doi: 10.1371/journal. pone. 0076289 .

25. Shirai Y, Yoshiji H, Noguchi R, Kaji K, Aihara Y, Douhara A, et al. Cross talk between toll-like receptor-4 signaling and angiotensin-II in liver fibrosis development in the rat model of non-alcoholic steatohepatitis. J Gastroenterol Hepatol 2013;28:723-30. doi: 10.1111/jgh.12112.

26. Kaji K, Yoshiji H, Kitade M, Ikenaka Y, Noguchi R, Shirai $\mathrm{Y}$, et al. Combination treatment of angiotensin II type I receptor blocker and new oral iron chelator attenuates progression of nonalcoholic steatohepatitis in rats. Am J Physiol Gastrointest Liver Physiol 2011;300:G1094-104. doi: 10.1152/ajpgi.00365.2010.

27. Noguchi R, Yoshiji H, Ikenaka Y, Kaji K, Aihara Y, Shirai $\mathrm{Y}$, et al. Dual blockade of angiotensin-II and aldosterone suppresses the progression of a non-diabetic rat model of steatohepatitis. Hepatol Res 2013;43:765-74. doi: 10.1111/hepr.12008.

28. Torres DM, Jones FJ, Shaw JC, Williams CD, Ward JA, Harrison SA. Rosiglitazone versus rosiglitazone and metformin versus rosiglitazone and losartan in the treatment of nonalcoholic steatohepatitis in humans: a 12-month randomized, prospective, open- label trial. Hepatology 2011;54:1631-9. doi: 10.1002/hep.24558.

29. Yokohama S, Yoneda M, Haneda M, Okamoto S, Okada
M, Aso K, et al. Therapeutic efficacy of an angiotensin II receptor antagonist in patients with nonalcoholic steatohepatitis. Hepatology 2004;40:1222-5. doi: 10.1002/ hep. 20420

30. Assali A, Ghayour-Mobarhan M, Sahebkar A, Hassani M, Kasaian J, Tatari F, et al. Association of angiotensin II type 1 receptor gene A1166C polymorphism with the presence of diabetes mellitus and metabolic syndrome in patients with documented coronary artery disease. Eur J Intern Med 2011;22:254-61. doi: 10.1016/j. ejim.2010.11.014.

31. Palatini P, Ceolotto G, Dorigatti F, Mos L, Santonastaso $\mathrm{M}$, Bratti $\mathrm{P}$, et al. Angiotensin II type 1 receptor gene polymorphism predicts development of hypertension and metabolic syndrome. Am J Hypertens 2009;22:208-14. doi: 10.1038/ajh.2008.319.

32. Abdollahi MR, Lewis RM, Gaunt TR, Cumming DV, Rodriguez S, Rose-Zerilli M, et al. Quantitated transcript haplotypes (QTH) of AGTR1, reduced abundance of mRNA haplotypes containing 1166C (rs5186:A>C), and relevance to metabolic syndrome traits. Hum Mutat 2007;28:365-73. doi: 10.1002/humu.20454

33. Procopciuc LM, Sitar-Tăut A, Pop D, Sitar-Tăut DA, Olteanu I, Zdrenghea D. Renin angiotensin system polymorphisms in patients with metabolic syndrome (MetS). Eur J Intern Med 2010;21:414-8. doi: 10.1016/j. ejim.2010.06.001.

34. Akasaka H, Katsuya T, Saitoh S, Sugimoto K, Fu Y, Takagi S, et al. Effects of angiotensin II type 1 receptor gene polymorphisms on insulin resistance in a Japanese general population: the Tanno-Sobetsu study. Hypertens Res 2006;29:961-7. doi: 10.1291/hypres.29.961.

35. Lonardo A, Lombardini S, Ricchi M, Scaglioni F, Loria P. Review article: hepatic steatosis and insulin resistance. Aliment Pharmacol Ther 2005;22 Suppl 2:64-70. doi: 10.1111/j.1365-2036.2005.02600.x.

36. Funahashi T, Matsuzawa Y. Adiponectin and the cardiometabolic syndrome: an epidemiological perspective. Best Pract Res Clin Endocrinol Metab 2014;28:93-106. doi: 10.1016/j.beem.2013.09.002.

37. Wu ZJ, Cheng YJ, Gu WJ, Aung LH. Adiponectin is associated with increased mortality in patients with already established cardiovascular disease: a systematic review and meta-analysis. Metabolism 2014;63:1157-66. doi: 10.1016/j.metabol.2014.05.001.

38. Pagano C, Soardo G, Esposito W, Fallo F, Basan L, Donnini $\mathrm{D}$, et al. Plasma adiponectin is decreased in nonalcoholic fatty liver disease. Eur J Endocrinol 2005;152:113-8.

39. Furuhashi M, Ura N, Higashiura K, Murakami H, Tanaka $\mathrm{M}$, Moniwa N, et al. Blockade of the renin-angiotensin system increases adiponectin concentrations in patients with essential hypertension. Hypertension 2003;42:7681. doi: 10.1161/01.HYP.0000078490.59735.6E.

40. Kiliszek M, Burzyńska B, Styczyński G, Maciag M, Rabczenko D, Opolski G. A1166C polymorphism of the angiotensin AT1 receptor (AT1R) gene alters endo- 
thelial response to statin treatment. Clin Chem Lab Med 2007;45:839-42. doi: 10.1515/CCLM.2007.151.

41. Bril F, Portillo Sanchez P, Lomonaco R, Orsak B, Hecht J, Tio F, et al. Liver Safety of Statins in Prediabetes or T2DM and Nonalcoholic Steatohepatitis: Post Hoc Analysis of a Randomized Trial. J Clin Endocrinol Metab 2017;102:2950-2961. doi: 10.1210/jc.2017-00867.

42. Eslami L, Merat S, Malekzadeh R, Nasseri-Moghaddam $\mathrm{S}$, Aramin H. Statins for non-alcoholic fatty liver disease and non-alcoholic steatohepatitis. Cochrane Database Syst Rev 2013;12:CD008623. doi: 10.1002/14651858. CD008623.

43. Eshraghian A. Current and emerging pharmacological therapy for non-alcoholic fatty liver disease. World $J$ Gastroenterol 2017;23:7495-7504. doi: 10.3748/wjg. v23.i42.7495. 